\title{
Derivation and validation of an accurate estimation of CD4 counts from the absolute lymphocyte count in virologically suppressed and immunologically reconstituted HIV infected adults
}

\author{
Barnaby Young ${ }^{1 *}$, Oon Tek $\mathrm{Ng}^{1,2}$, David Chien Lye ${ }^{1,3}$ and Yee Sin Leo ${ }^{1,2}$
}

\begin{abstract}
Background: A simple method to estimate CD4 counts in stable, HIV infected virologically-suppressed and immune-reconstituted adults could save the expense of unnecessary formal testing.

Methods: Using a baseline CD4 percent, CD4 counts were estimated from subsequent absolute lymphocyte counts (ALC) measured by an automated FBC machine (CD4 estimate calculated by the ALC multiplied by the baseline CD4 percent). The accuracy of this approach was established in a large, retrospective clinical laboratory dataset of virologically-suppressed HIV infected subjects. A case-control study explored important clinical factors for accurate estimates, and a heuristic algorithm was derived and validated in a random sample.

Results: Data from 3,630 subjects were available. CD4 counts were generally accurately estimated, with a mean $6.1 \%$ underestimation. Overall $83.3 \%$ of CD4 estimates were within $25 \%$ of the actual values, with $12.1 \%$ CD4 counts underestimated by more than $25 \%$, and $4.5 \%$ overestimated. The CD4 count was increasingly underestimated with time from baseline, and the degree of underestimation correlated with baseline CD4 percent $(p<0.0001)$. From the case-control study, baseline CD4 percent of $\geq 20$, no illness requiring hospitalization and more than a year since starting or switch of anti-retroviral therapy were identified as significant predictors of inaccurate estimates. Employing this simple algorithm, CD4 estimate accuracy improved to a mean $1.3 \%$ underestimation, and the proportion of estimates within $25 \%$ of the actual value increased to $93.4 \%$.

Conclusions: In virologically-suppressed and immune-reconstituted HIV-infected adults, the CD4 count can be accurately estimated from the ALC using a baseline CD4 percent for at least 2 years after measurement.
\end{abstract}

\section{Background}

The clinical value of routinely monitoring CD4 counts in HIV-infected adults with virologic suppression and immune reconstitution is questionable. After counts have risen to more than $300-350$ cells $/ \mathrm{mm}^{3}$, the proportion subsequently falling to less than 200 cells $/ \mathrm{mm}^{3}$ is reported as only $1.1-2.9 \%$ in retrospective studies [1-4]. The

\footnotetext{
*Correspondence: Barnaby_young@ttsh.com.sg

'Institute of Infectious Diseases and Epidemiology, , Tan Tock Seng Hospital, Singapore, Singapore

Full list of author information is available at the end of the article
}

majority of these declines are predictable, transient, not directly due to HIV or anti-retroviral therapy (ART) and do not increase the risk of opportunistic infections. Reducing the frequency of CD4 testing has the potential for substantial cost savings - estimated at up to $\$ 18.1$ million per year in the US [5]. Based on these findings, recent guidelines from the International AIDS Society (IAS) have recommended that among virologically suppressed individuals with CD4 counts above 500 cells $/ \mathrm{mm}^{3}$ further monitoring of CD4 counts is optional [6]. Yet while patients may feel anxious over clinically insignificant 
fluctuations in the CD4 count, resistance to reduced monitoring has also been suggested.

We hypothesised that following virologic suppression and immune reconstitution, CD4 counts could be estimated using lymphocyte measurements from an automated full blood count (FBC) analyser. Such a method has not, to our knowledge, been reported before. FBCs are an important part of routine HIV care regardless of disease stage. In a retrospective cohort study, we aimed to derive and validate a simple heuristic algorithm that is able to estimate CD4 counts reliably.

\section{Methods}

All available HIV viral loads (VL) and CD4 panels (including CD4/8 absolute values, percent and lymphocyte counts) were extracted from an electronic laboratory database. Where at least one CD4 or VL was available for a subject, absolute lymphocyte counts (ALCs) from FBC measurements were also extracted. Data was available from January 2008 to September 2013. CD4 panels were performed on a flow cytometer (BD Biosciences, New Jersey, USA), while FBC results were from an automated analyser (Beckman Coulter, California, USA). No patient information was available from this database.

Periods of virologic suppression were identified. Virologic suppression was defined as at least two consecutive viral loads less than 200 copies $/ \mathrm{ml}$ within 400 days of each other, similar to other studies. During each period of virologic suppression, the first absolute CD4 measurement greater than 300 cells $/ \mathrm{mm}^{3}$ and the accompanying CD4 percent was identified and used as baseline. For the following 720 days, CD4 and ALC measurements taken on the same day were collected. The baseline CD4 percent was used to estimate an absolute CD4 count from subsequent ALCs (CD4 estimate $=$ ALC $\mathrm{x}$ baseline CD4 percent). Estimated $\mathrm{CD} 4$ values were compared with the actual value for accuracy (predicted CD4/actual CD4 $\mathrm{x}$ 100). Two factors were chosen as clinically relevant markers of accuracy:

1. Proportion of CD4 estimates within $10 \%$ and $25 \%$ of the actual value

2. Percentage deviation of accuracy from zero covering $90 \%$ of estimates

Actual CD4 values less than 200 cells $/ \mathrm{mm}^{3}$ were identified. Electronic and paper patient records were reviewed to determine if a cause could be identified - including serious illness requiring hospital admission within one month before CD4 measurement, cytotoxic chemotherapy, hepatitis $\mathrm{C}$ treatment or any other expected cause of lymphopenia.

A case-control study (1:2) of 150 individuals was then performed to identify factors predicting inaccurate results. Cases were defined as greater than $25 \%$ underestimation of the actual CD4 value, while controls as estimates within $25 \%$.

Cases and controls were matched by expected confounders: baseline CD4 percent (exact) and days from baseline to CD4 estimate (within 28 days). Cases and controls were sampled randomly without replacement, each subject contributing only one estimate. Electronic and paper records were reviewed and compared for demographics, history of AIDS-defining infections, trough CD4 less than 200 cells $/ \mathrm{mm}^{3}$, ART regimen and serious illnesses within one month prior to the date of CD4 or ALC testing.

Results from the case-control study were used to determine simple baseline clinical requirements which were predictive of accurate CD4 estimates. The algorithm was validated in a sample of 100 individuals by reviewing electronic and paper records. Where possible new CD4 estimates were generated when baseline clinical conditions were not met. Accuracy of estimates was compared between the two sets.

Statistical analysis was performed using R [7]. All samples were assumed to be independent (including for the case-control study), and p-values were interpreted with a two-tailed significance level of $5 \%$.

This study was performed at the Communicable Disease Centre (CDC), Tan Tock Seng Hospital Singapore. $\mathrm{CDC}$ is the main treatment centre for HIV in Singapore, with approximately 2,500 patients attending for HIV care annually. The study was approved by the National Healthcare Group Domain Specific Review Board, and access to the database approved by the Head of Infectious Diseases Department, Tan Tock Seng Hospital.

\section{Results}

Fifteen thousand seventy-nine HIV VLs and 29,927 CD4 counts were available for analysis from 3,630 subjects. 3,885 paired CD4-ALCs covering 1,444 periods of virologic suppression in 1,388 subjects met the inclusion criteria for the study. Median frequency of VLs was 175 days (inter-quartile range (IQR): 127-245), median frequency of CD4 counts was 147 days (IQR: 105-178).

\section{Estimating CD4 values}

Overall $83.3 \%$ of CD4 estimates were within $25 \%$ of the actual values, and $41.3 \%$ within $10 \% .90 \%$ of predicted CD4 counts were within $30 \%$ of the actual value. Accuracy measures approximated the normal distribution with a mean $6.1 \%$ underestimation. As a result, while $12.1 \%$ CD4 counts were underestimated by more than $25 \%$, only $4.5 \%$ were overestimated.

Lymphocyte counts correlated well between flow cytometry and automated FBC measurements $(r=0.970$, $\mathrm{p}<0.0001$ ). A median absolute difference of $5.5 \%$ was 
observed, but without significant variation of the mean from zero (one sample $t$ test, $p=0.40$ ). No significant difference between lymphocyte counts measured by CD4 panel or FBC were identified when results were stratified by absolute $\mathrm{CD} 4$ count or $\mathrm{CD} 4$ percent.

The ALC was only moderately correlated with the CD4 count $(\mathrm{r}=0.589, \mathrm{p}<0.0001)$, and correlation with the $\mathrm{CD} 4 \%$ was negative and substantially weaker $(r=-0.216$, $\mathrm{p}<0.0001$ ). Thus while the ALC is an accurate measure of lymphocyte counts, its ability to predict the CD4 count is limited.

As expected, absolute CD4 count and CD4 percent were significantly higher with increasing time from baseline measurement $(\mathrm{p}<0.0001)$. CD4 count was increasingly underestimated with time, and the degree of underestimation correlated with baseline CD4 percent $(\mathrm{p}<0.0001)$. When the baseline CD4 percent was $\geq 25$, more than $90 \%$ of estimates were within $25 \%$ of the actual value out to 720 days (Fig. 1).

\section{Case-control study}

One hundred controls were matched to 50 cases. Case and controls were well matched by $\mathrm{CD} 4$ percent baseline and days (Table 1).

No significant differences in baseline lymphocyte counts, absolute $\mathrm{CD} 4, \mathrm{CD} 8$ or $\mathrm{CD} 4 / 8$ ratio were identified between cases and controls. There was also no significant difference between lymphocyte measurements by FBC and flow cytometry.

Controls had a significantly smaller increase in CD4 percent from baseline to estimate $(\mathrm{p}<0.0001)$, and significantly lower CD4 counts $(p=0.002)$. Stable CD4 percent with time is clearly important for accurate results, and stability was not affected by age, gender, history of AIDS-defining illnesses or a documented CD4 trough of less than 200 cells $/ \mathrm{mm}^{3}$. However, presence of serious illness requiring hospital admission predicted inaccurate estimates $(p=0.0001)$, as did less than a year since starting ART $(p=0.035)$. Change of ART in virologically suppressed hosts was common and more frequent among cases, but this rate were not statistically significantly different from controls. 15/17 (88.2 \%) of switches were between nucleoside reverse transcriptase inhibitors, primarily from stavudine to zidovudine or tenofovir.

\section{Heuristic algorithm and validation}

The following baseline parameters for CD4 estimates were chosen for the validation phase of the study:

1. Baseline CD4 percent $\geq 20$, Absolute CD $4 \geq 300$

2. Virologic suppression $(<200$ copies/ml)

3. More than one year since starting ART or a change in regimen

4. No serious concomitant illness requiring hospital admission or expected to cause lymphopenia

A new sample of 100 individuals was screened following the above algorithm, yielding 292 CD4 estimates. 5 subjects were excluded from validation as no results where available meeting baseline criteria, while 49 subjects required recalculation of estimates. Primary reasons for recalculation were ART related - treatment for less than one year in 28 (51.9\%) and switch of therapy in 22 (40.7 \%). 243 CD4 estimates were analysed after validation. There was no statistically significant difference in the average time since baseline $\mathrm{CD} 4 \%$ in either group

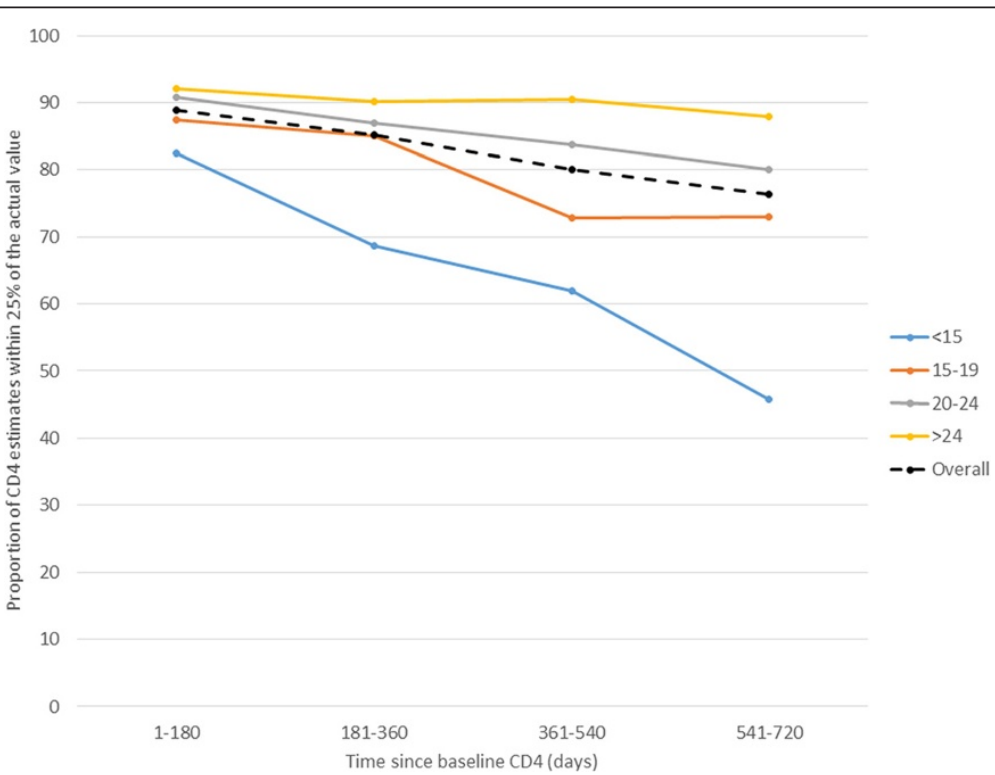

Fig. 1 Accuracy of CD4 estimates as a function of time and stratified by baseline CD4 percent. 'Overall' includes all results 
Table 1 Matched case-control study

\begin{tabular}{|c|c|c|c|}
\hline & $\begin{array}{l}\text { Case } \\
(n=50)\end{array}$ & $\begin{array}{l}\text { Controls } \\
(n=100)\end{array}$ & Odds Ratio, p-value \\
\hline Baseline CD4\% (mean) & 19 & 19 & $N S^{a}$ \\
\hline $\begin{array}{l}\text { Days from baseline CD4\% } \\
\text { to estimated CD4 (mean) }\end{array}$ & 393 & 393 & $N S^{a}$ \\
\hline Mean age (years) & 45 & 47 & $N S^{a}$ \\
\hline Proportion $>50$ years & $34 \%$ & $38 \%$ & $0.85(0.46-1.55), N^{b}$ \\
\hline Sex (male) & $90 \%$ & $90 \%$ & $1(0.35-2.84), \mathrm{NS}^{\mathrm{b}}$ \\
\hline $\begin{array}{l}\text { History of AIDS defining } \\
\text { illness or trough } C D 4<200\end{array}$ & $62 \%$ & $66 \%$ & $0.83(0.44-1.57), \mathrm{NS}^{\mathrm{b}}$ \\
\hline First line ART & $84 \%$ & $88 \%$ & $0.71(0.28-1.73), N^{b}$ \\
\hline Days ART (mean) & 795 & 1004 & $N S^{a}$ \\
\hline$<180$ days & $38 \%$ & $21 \%$ & $2.30(1.09-4.87), 0.032^{b}$ \\
\hline$<360$ days & $50 \%$ & $32 \%$ & $2.12(1.06-4.26), 0.035^{b}$ \\
\hline$<720$ days & $62 \%$ & $45 \%$ & $1.99(1.00-3.99), 0.058^{b}$ \\
\hline $\begin{array}{l}\text { ART switch (not due to } \\
\text { virologic failure) }\end{array}$ & $18 \%$ & $8 \%$ & $2.52(0.91-7.01), 0.1^{b}$ \\
\hline Illness & $16 \%$ & $0 \%$ & $0.0001^{b}$ \\
\hline
\end{tabular}

${ }^{\mathrm{a}}$ students $t$ test, ${ }^{\mathrm{b}}$ Chi square, Fischer's exact

(derivation 352 days vs. validation 359 days), while mean baseline CD4 percent increased marginally from 25.4 to $26.8 \%(p=0.002, t$-test $)$.

The algorithm significantly improved accuracy of CD4 estimates. The proportion of estimates within $25 \%$ of the actual value increased from 86.0 to $93.4 \%(p=0.007)$, and within $10 \%$ from 46.6 to $57.2 \%(p=0.015) .90 \%$ confidence intervals for estimates narrowed from 28 to $22 \%$.

Estimates were significantly more accurate in the first versus second year for the derivation cohort (mean $1.8 \%$ vs. $6.2 \%$ underestimation, $p=0.044$ ). This narrowed after validation (mean $0 \% \mathrm{v} 4.7 \%$ underestimation, $p=0.002$ ).

\section{CD4 dips to less than 200 cell $/ \mathrm{mm}^{3}$}

From the initial cohort, 49 (1.3\%) CD4 counts from 41 subjects were less than 200 cells $/ \mathrm{mm}^{3}$. 34 (69.3\%) were identified in the first year of care. 31/49 of these were also predicted by the algorithm (sensitivity $63.3 \%, 95 \%$ CI: 48.3-76.6; specificity $98.8 \%, 95 \%$ CI 98.4-99.1). After reviewing the charts of the 41 subjects, serious illnesses were identified in 10. CD4 dips were typically transient, in only 6 of 39 subjects (15.4\%) with follow up CD4 counts were dips below 200 cells $/ \mathrm{mm}^{3}$ sustained for more than one CD4 measurement. No AIDSdefining opportunistic infections were diagnosed when the CD4 count was less than 200 cells $/ \mathrm{mm}^{3}$.

\section{Discussion}

In immune-reconstituted, virologically suppressed and clinically stable HIV-infected adults, the CD4 count can be accurately estimated by multiplying the absolute lymphocyte count by a baseline CD4 percent. This estimate is within $25 \%$ of the actual value almost $95 \%$ of the time for at least the first two years from baseline measurement.

Previous studies have demonstrated that using ALC cutoffs to predict an absolute CD4 count of $<200$ cells $/ \mathrm{mm}^{3}$ or response to ART is unreliable for clinical use $[7,8]$. The primary reason for this lack of concordance between ALC and CD4 is variability in the CD4 percent rather than large discrepancies between lymphocyte counts when measured by an automated FBC analyser or lymphocyte subset flow cytometer. Stability of the CD4 percent is crucial for accurate CD4 estimates using the method outlined here. The CD4 percent has less physiological variability than the absolute count, and following immune reconstitution increases in the CD4 percentage are proportionally smaller [9].

Data from this study supports reducing the frequency of CD4 monitoring in clinically stable, virologicallysuppressed and immune-reconstituted adults. Similar to previous studies, the proportion of CD4 values less than 200 cells $/ \mathrm{mm}^{3}$ was low - only $1.3 \%$ and was generally predictable, transient and not associated with opportunistic infections. The clinical utility of CD4 counts in this population is limited, yet, measurement continues. We propose that where patients or healthcare providers prefer knowing a CD4 value, this study provides a simple and accurate method of estimating it. The reassurance of this estimate may facilitate implementation of recent guidelines to reduce the frequency of testing and achieve anticipated cost savings. At our institution, CD4 panels are substantially more expensive than FBC counts (113.42 vs 25.74 SGD, before government subsidies). For the 100 patients in the validation phase of this study, stopping CD4 counts was equivalent to a cost saving of $\$ 27,561$ over two years.

This study has several limitations. It is retrospective and was performed in a population that was largely male with a low incidence of intravenous drug use and hepatitis C infection (2\%) [10]. Zidovudine or stavudinebased anti-retroviral therapy were standard first line treatments until recently, and the association of these agents with lymphopenia may reduce the accuracy of this method. A VL periodicity of 400 days was chosen to reflect local clinical practice, where annual testing is sometimes necessary due to cost considerations. Inevitably this does not guarantee continuous virologic suppression. Treatment interruptions which were unrecognised or not confirmed virologically were not excluded from this analysis. The number of $\mathrm{CD} 4$ estimates per individual was not restricted. However, more frequent monitoring is more likely during periods of clinical instability or early during the treatment course. Both limitations are likely to 
bias this study towards inaccurate CD4 estimates and are mitigated by the validation phase.

An estimated CD4 count within $25 \%$ of the actual value was chosen a priori as clinically adequate. What degree of accuracy is acceptable is debatable, but this level is similar to expected physiological variability [9, 11]. Finally, a single set of simple clinical rules was chosen for validation in a relatively small cohort which may not have been representative. These rules may not be the best possible for accurate CD4 estimates.

\section{Conclusion}

In virologically-suppressed and immune-reconstituted HIV-infected adults, the CD4 count can be accurately estimated from the ALC using a baseline CD4 percent for at least 2 years after measurement.

\section{Competing interests}

All authors have no conflicts of interest to declare, and received no funding for this article.

\section{Authors' contributions}

BY conceived and designed the study, acquired and analysed the data. OTN conceived the study and acquired the data, DL and YSL conceived the study. All authors were involved in drafting or revising the manuscript. All authors read and approved the final manuscript.

\section{Acknowledgments}

Thanks to Ms Chin Mei Ng for assistance with database management.

\section{Meetings}

Findings from this study were presented in part at HIV Drug Therapy Glasgow (2-6 November 2014).

\section{Author details}

${ }^{1}$ Institute of Infectious Diseases and Epidemiology, , Tan Tock Seng Hospital, Singapore, Singapore. ${ }^{2}$ Saw Swee Hock School of Public Health, National University of Singapore, Singapore, Singapore. ${ }^{3}$ Yong Yoo Lin School of Medicine, National University of Singapore, Singapore, Singapore.

Received: 14 May 2015 Accepted: 3 August 2015

Published online: 13 August 2015

\section{References}

1. Gale HB, Gitterman SR, Hoffman HJ, Gordin FM, Benator DA, Labriola AM, et al. Is frequent CD4+ T-lymphocyte count monitoring necessary for persons with counts $>=300$ cells/muL and HIV-1 suppression? Clin Infect Dis. 2013;56(9):1340-3.

2. Girard PM, Nelson M, Mohammed P, Hill A, van Delft Y, Moecklinghoff C. Can we stop CD4+ testing in patients with HIV-1 RNA suppression on antiretroviral treatment? Aids. 2013;27(17):2759-63.

3. Whitlock GG, Ahmed N, Benn P, Edwards S, Waters L. Stop routine CD4 monitoring in HIV-infected patients with fully suppressed virus and CD4>=350 cells/ml. Clin Infect Dis. 2013;57(2):327-8.

4. Ford N, Stinson K, Davies MA, Cox V, Patten G, Cragg C, et al. Is it safe to drop CD4+ monitoring among virologically suppressed patients: a cohort evaluation from Khayelitsha, South Africa. Aids. 2014;28(14):2003-5.

5. Hyle EP, Sax PE, Walensky RP. Potential savings by reduced CD4 monitoring in stable patients with HIV receiving antiretroviral therapy. JAMA Intern Med. 2013;173(18):1746-8.

6. Gunthard HF, Aberg JA, Eron JJ, Hoy JF, Telenti A, Benson CA, et al. Antiretroviral treatment of adult HIV infection: 2014 recommendations of the International Antiviral Society-USA Panel. Jama. 2014:312(4):410-25.

7. Bagchi S, Kempf MC, Westfall AO, Maherya A, Willig J, Saag MS. Can routine clinical markers be used longitudinally to monitor antiretroviral therapy success in resource-limited settings? Clin Infect Dis. 2007;44(1):135-8.
8. Sreenivasan S, Dasegowda V. Comparing absolute lymphocyte count to total lymphocyte count, as a CD4 T cell surrogate, to initiate antiretroviral therapy. J Glob Infect Dis. 2011;3(3):265-8.

9. Raboud JM, Haley L, Montaner JS, Murphy C, Januszewska M, Schechter MT. Quantification of the variation due to laboratory and physiologic sources in CD4 lymphocyte counts of clinically stable HIV-infected individuals. J Acquir Immune Defic Syndr Hum Retrovirol. 1995;10 Suppl 2:S67-73.

10. Lim RB, Tan MT, Young B, Lee CC, Leo YS, Chua A, et al. Risk factors and time-trends of cytomegalovirus (CMV), syphilis, toxoplasmosis and viral hepatitis infection and seroprevalence in human immunodeficiency virus (HIV) infected patients. Ann Acad Med Singapore. 2013;42(12):667-73.

11. Malone JL, Simms TE, Gray GC, Wagner KF, Burge JR, Burke DS. Sources of variability in repeated T-helper lymphocyte counts from human immunodeficiency virus type 1-infected patients: total lymphocyte count fluctuations and diurnal cycle are important. J Acquir Immune Defic Syndr. 1990;3(2):144-51.

\section{Submit your next manuscript to BioMed Central and take full advantage of:}

- Convenient online submission

- Thorough peer review

- No space constraints or color figure charges

- Immediate publication on acceptance

- Inclusion in PubMed, CAS, Scopus and Google Scholar

- Research which is freely available for redistribution 